\title{
The effects of categorization and categorical repetition on successive recalls of word lists
}

WILLIAM A. ROBERTS AND INA SMITH

VASSAR COLLEGE

The hypothesis that Ss develop a set to organize words by categories which facilitates recall was tested by measuring successive recall under conditions of high and low listcategorization. High categorization led to improvement in recall while low categorization did not, but the improvement under the high categorization condition appeared only when different categories were used in each list. When the same categories appeared on each list, high categorization produced no better performance than low categorization, and this finding was attributed to proactive interference.

In a number of experiments, it has been demonstrated that Ss who learn word lists divided into conceptual categories will recall the words in category clusters although the words were presented randomly. In an experiment by Bousfield \& Cohen (1956), it was found that Ss who had had previous experience in recalling a categorized list showed better recall and clustering with a second list than Ss who had not had prior experience. Bousfield and Cohen interpreted this finding in terms of the development of a set to look for categories which aided organization and recall of the material. The increased recall found in the group with previous training cannot, however, be unequivocally attributed to the development of a set to organize by categories since this result may reflect only an increased general ability to learn and recall. The hypothesis that a set to organize words into categories can develop and facilitate recall is here tested by measuring successive recall under varying degrees of categorization. If this hypothesis is correct, improvement in recall should be greater where the possibility of organization into categories is high than where it is low. One other variable, categorical repetition, was also studied in this experiment.

Method

Four groups of Ss learned and recalled 10 different lists of words under massed practice. Two groups learned 20-word lists divided into four categories of five words each. The other two groups learned 20-word lists divided into 20 categories, one word in each category. At each level of categorization, one group learned lists containing the same categories throughout, while for the other group, the categories were different in each of the 10 lists. Thus, the four groups were (1) a four-category group with the same four categories in each list (4 Cat-SC), (2) a four-category group with four different categories in each list (4 Cat-DC), (3) a 20-category group with the same 20 categories in each list (20 Cat-SC), and (4) a 20-category group with 20 different categories in each list (20 Cat-DC). In the overall design, then, degree of categorization and categorical repetition were varied in a 2 by 2 factorial manner as between-Ss factors while recall was a within-Ss factor.

Each list of words used was made up of nouns drawn from the Thorndike and Lorge word count (1944), and the mean frequency of occurrence of the words in each list was approximately 25 per million. The words in each list were randomly ordered for presentation to the Ss. As an example of the categories used, the 4 Cat-SC Group learned lists composed of nouns from the categories of animals, occupations, countries, and first names. To form the lists for the 4 Cat-DC, 20 Cat-SC, and 20 Cat-DC groups, nouns were drawn from these and additional categories.

The Ss were 32 women students enrolled in introductory psychology and child study courses at Vassar College. Each of the four groups contained eight Ss, and, in order to observe clearly the transfer effects over recalls, the groups were matched for mean number of words recalled on the first recall. Within each group, list order was balanced over Ss so that no two lists were presented at the same recall position.

When $\mathrm{S}$ entered the laboratory, she was told that she would be read 10 lists of words and would be given a test of free recall after each list. $S$ was asked to memorize as many of the words as possible when they were read to her, and it was emphasized that she should recall the words in the order in which she remembered them and not necessarily in the order in which they were presented. The first list was read once to $S$, and she was then allowed $4 \mathrm{~min}$. to write down as many words as she could remember. After the $4 \mathrm{~min}$. recall period had elapsed, the same procedure was followed with the second list and so on until 10 lists had been recalled. Results and Discussion

Figure 1 presents the mean words recalled as a function of recalls for each group. Early and marked improvement in recall appears in the 4 Cat-DC Group, but no improvement over recalls is apparent for the other three groups. In fact, there seems to be a slight decline in the performance of groups 4 Cat-SC and 20 Cat-DC. Analysis of variance on words recalled shows a significant Categorization-effect $(F=8.89$, $\mathrm{df}=1 / 28)$, a significant Categorical Repetition-effect ( $F=4.77$, df $=1 / 28$ ), and a significant Categorization by Categorical Repetition interaction $(F=17.27, d f=1 / 28)$. Recalls is not a significant effect, and none of the two-factor interactions with Recalls is significant. However, the triple 


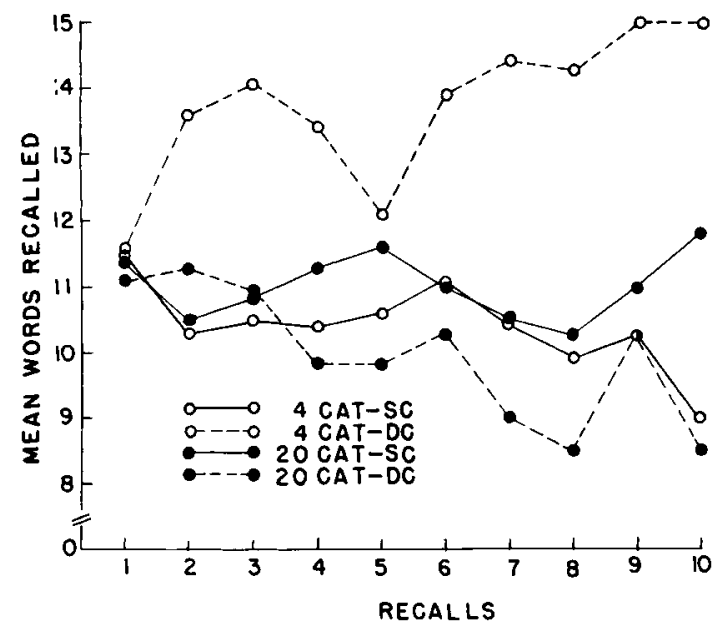

Fig. 1. Mean words recalled as a function of recalls.

interaction of Recalls by Categorization by Categorical Repetition is significant ( $F=2.87, d f=9 / 252$ ).

In accord with the organizational set hypothesis, a high degree of categorization (four categories) leads to positive transfer over recalls. This effect, however, appears only when there is no categorical repetition. With categorical repetition, a high degree of cate-

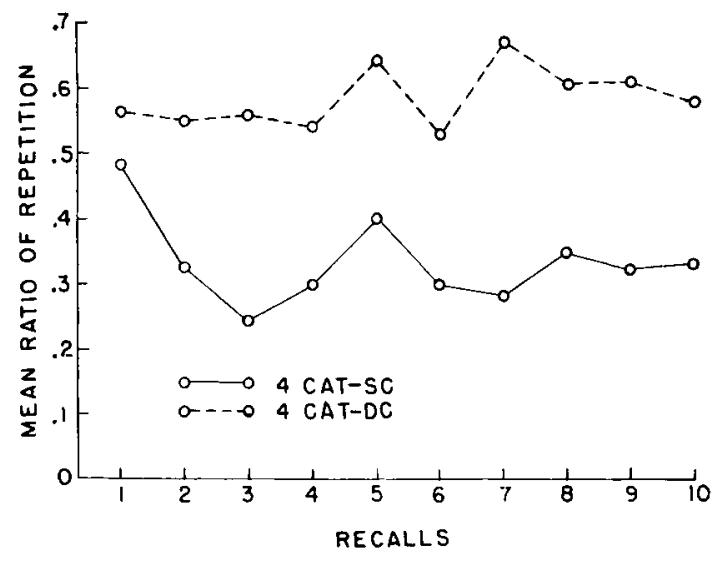

Fig. 2. Mean ratio of repetition as a function of recalls. gorization produces no better performance than a low degree of categorization (20 categories). In order to look at the effects of categorical repetition on intralist organization, the ratio of repetition was calculated for each $S$ in the 4-category groups at each recall. The ratio of repetition was determined by dividing the number of sequential categorical repetitions by the number of words recalled minus one. (Categorical intrusion errors were included in the determination of the ratio of repetition.) The mean ratio of repetition is plotted as a function of recalls for each group in Fig. 2. Both curves begin at about the same level, but the 4 Cat-DC curve remains at the initial level over all 10 recalls while the 4 Cat-SC curve declines rapidly to a lower level and remains there. Analysis of Variance on these curves reveals a significant Groups-effect $(F=14.75$, $\mathrm{df}=1 / 14$ ), but the Recalls-effect and the Groups by Recalls interaction are not significant. Thus, the occurrence of the same categories on successive lists leads to a significant dis ruption of intralist organization.

The disruption of intralist organization and the corresponding failure of improvement in recall found for the 4 Cat-SC Group can be accounted for in terms of proactive interference arising from similar words which appeared on prior lists. From this account, it might be expected that more categorical intrusion errors would appear for the 4 Cat-SC Group than for the 4 Cat-DC Group. However, the total categorical intrusion errors over all recalls are 45 for the 4 Cat-DC Group and 30 for the 4 Cat-SC Group. While no evidence of differential interference is found in the categorical intrusion error data, it still seems quite probable that the poorer performance of the 4 Cat-SC Group was the product of interlist confusion. Most probably, Ss in the 4 Cat-SC Group remembered many words from both the justpresented list and prior lists which they did not write down because they could not identify their list membership. This would lead to a low number of both correct recalls and categorical intrusion errors.

\section{References}

Bousfield, W. A., \& Cohen, B. H. Clustering in recall as a function of the number of word-categories in stimulus-word lists. J. gen. Psychol., 1956, 54, 95-106.

Thorndike, E. L., \& Lorge, I. The teacher's word book of 30,000 words. New York: Teachers College, Columbia University, 1944. 